\title{
The natural satellites ephemerides facility MULTI-SAT
}

\author{
N. V. Emel'yanov ${ }^{1,2}$ and J.-E. Arlot ${ }^{1}$ \\ 1 Institut de mécanique céleste et de calcul des éphémérides - Observatoire de Paris, UMR 8028 du CNRS, \\ 77 avenue Denfert-Rochereau, 75014 Paris, France \\ e-mail: arlot@imcce.fr \\ 2 Sternberg astronomical institute, 13 Universitetskij prospect, 119992 Moscow, Russia \\ e-mail: emelia@sai.msu.ru
}

Received 17 March 2008 / Accepted 20 May 2008

\section{ABSTRACT}

\begin{abstract}
Context. There is a need in some research facilities for deriving ephemerides, controlling observations, verifying various models of motion, and calculating the coordinates in space of natural planetary satellites.

Aims. The goal of our work is to elaborate the ephemerides of all natural satellites based upon all observations available to date and readily accessible for any user via the Internet.

Methods. For all outer planetary satellites, original numerical models of motion are used that are based on all published observations. For other satellites, the theoretical models of the motion are taken from publications that are as recent as possible. Complete collection of the theories and models of motion is realized as a software for the ephemerides of natural satellites available on the web pages of the so-called server MULTI-SAT.

Results. A new facility for producing ephemerides of all natural satellites of planets (except the Moon) has been created at IMCCE and SAI. Special features of the ephemerides are realized, such as predicting the phenomena and providing configurations useful for the observers. The server MULTI-SAT is accessible through the Internet. The URL addresses are http://www .imcce.fr/sat (English and French versions at IMCCE) and http://www.sai.msu.ru/neb/nss/index.htm (English, French, and Russian versions at SAI). This paper includes a complete review of the most precise theories of motion of all natural satellites that we used and an analysis of the precision of the proposed ephemerides.
\end{abstract}

Key words. ephemerides - planets and satellites: general

\section{Introduction}

Many institutes in the world are involved in studies of the natural satellites of planets. Researchers use basically the same data for their work and similar software tools. Therefore, there is a need for some research facilities in the form of computing codes for deriving ephemerides, evaluating observations, verifying various models of motion, and calculating the coordinates of satellites for the analysis of observational data, as from space missions and ground-based observations. The software tools for calculating ephemerides are the final result of research, as they actually incorporate the complete knowledge about dynamics of the satellites of planets including all available observational data.

Developing the theories of motion of celestial bodies and determining their orbital parameters from observations are both quite complicated procedures. Various methods and observational data are used in them; therefore, it is reasonable to have several independent models of satellite motion in the world based upon observations and verified by cross checking. This would always allow one to have information on satellite motion, the most adequate information on satellite motion, based on observations and reliable ephemerides. For all these reasons, we developed a new facility for natural satellites ephemerides: the MULTI-SAT server.

\section{The history of the natural satellites ephemerides server}

At the beginning, ephemerides were provided through printed tables. The oldest ephemerides of the Galilean satellites consist in configurations providing a visual plot of the positions of the satellites around the planet. As theories of the motion of the satellites became more precise, it was necessary to provide more precise positions. Configurations are still published in the Astronomical Almanach, as in the Supplement to Connaissance des Temps and in most of the published ephemerides worldwide. Starting during the 20th century, tables of numbers were published allowing positions of the satellites to be calculated relative to the planet. The arrival of powerful computers made it possible to obtain and publish the expansions of the coordinates of satellites in Chebyshev series in mixed functions, enabling calculation of the ephemerides using electronic pocket calculators with an accuracy very close to the accuracy of the theory itself.

The dynamics of the natural planetary satellites is being studied in several laboratories worldwide, and the results of all these works have been published in international journals. However, most of the ephemerides deduced from theoretical works are not available. Very few laboratories provide formulas for calculating the satellite coordinates, so researchers must extract the data from the publications to make their own ephemerides. 
Some laboratories have a long history of providing ephemerides of the natural planetary satellites, and they now use the Internet facilities, making ephemerides online for the users. Such services were proposed by the Jet Propulsion Laboratory (JPL) for the need of NASA but have also been useful to many astronomers. Similarly in France, the IMCCE (formerly Bureau des Longitudes) has proposed ephemerides online for the need of the CNES (the French Space Agency), also useful for astronomers. Only those two Internet servers have proposed ephemerides for all the natural planetary satellites, together with ephemerides of the other bodies of the solar system. The MPC (Minor Planet Center) has recently proposed ephemerides of the irregular satellites of the giant planets since they are observed like asteroids. However, many other laboratories doing research in celestial mechanics have proposed their own accurate theoretical models for selected satellites. To use such models appeared to be very difficult, requiring the code source of the computer program.

Ephemerides software of natural satellites has been created and developed in 1995 in the Celestial Mechanics Department of Sternberg Astronomical Institute (SAI), Moscow University (Emel'yanov 1996a,b). The computer codes for calculating ephemerides are based on the most accurate theories for the motion of planet satellites.

Then, IMCCE in Paris and SAI in Moscow started to collaborate in the field of the natural planetary satellites databases and ephemerides. On the basis of the software developed in SAI, we decided to develop the server MULTI-SAT, providing a lot of services concerning the ephemerides of the natural satellites on the web-pages on the Internet. New original theoretical models created in IMCCE and SAI were fitted to the observations gathered in the Natural Satellites Data Base (Arlot \& Emel'yanov 2008). These models were included in the ephemerides server MULTI-SAT.

\section{The data provided by the ephemerides server MULTI-SAT}

The data are provided through several different webpages written in HTML language. On May 2, 2007, the 166 natural planetary satellites have ephemerides with accuracy that is sufficient for many purposes. Some theoretical models for the main satellites are made at IMCCE and at SAI. Original numerical models of motion of all outer satellites of planets are fitted to the observations in SAI as described in Emel'yanov (2005), Emel'yanov \& Kanter (2005), Emel'yanov (2007). Some theoretical models for the natural satellites were taken from publications. The address of the access page is http://www. imcce.fr/sat (French and English) or http://www.sai.msu.ru/neb/nss/index.htm (French, English and Russian). All systems of satellites are presented on this page for each planet. For Jupiter, it is split into inner satellites (including all satellites inside the orbit of Callisto including it) and into outer satellites (for satellites outside the orbit of Callisto).

Three possibilities are offered:

- calculations of a series of positions with a constant time step between them for given satellite;

- calculations of positions at any series of dates provided by the user including $(\mathrm{O}-\mathrm{C}) \mathrm{s}$ when observed positions are provided for these dates for a given satellite. Statistics on these observations $(\mathrm{rms}, \ldots)$ are also given;
- graphical configurations of each system of satellites centered on a chosen satellite or on the planet, together with the relative positions of the concerned satellites.

The two first ephemerides offer many possibilities for the calculated positions:

- right ascension and declination of the concerned satellite or of the planet or of the barycenter of the system in several reference frames (J2000, B1950, 1900, mean of the date, of the beginning of the year or apparent). All these reference frames have been used by observers and may be useful for calculating $(\mathrm{O}-\mathrm{C}) \mathrm{s}$;

- coordinates relative to any satellite or to the planet (in tangential or differential $X$ and $Y$; in separation and position angle; geocentric or topocentric);

- specific coordinates such as pseudo-heliocentric in order to calculate the position of the shadow of an object of the system (for $(\mathrm{O}-\mathrm{C}) \mathrm{s}$ when observing a phenomena when possible with the concerned planetary system);

- plantocentric coordinates of the Earth and the Sun, planetocentric synodic and heliocentric longitude and latitude of the satellite;

- for observers, the apparent distance satellite-Sun, the phase angle and the magnitude of the satellite;

- osculating Keplerian parameters. For theoreticians, planetocentric $X, Y, Z$ coordinates, together with velocities.

The theoretical model to be used may be chosen among the best ones that we have programmed. The planetary ephemeris may be chosen among INPOP, DE405, DE406, DE200, VSOP87. Any terrestrial site may be chosen through the IAU number of the observatory.

Besides the ephemerides for the positions presented above, MULTI-SAT provides ephemerides for phenomena or for specific configurations:

- times of maximum elongations of satellites for the preparation of observations; and

- times and other parameters of mutual occultations and eclipses of satellites or of eclipses of satellites by the planet, which may be useful for preparating observational campaigns.

The search for phenomena for the satellites of Jupiter, Saturn, and Uranus is being carried out by users running a special software from webpages.

The eclipses by Jupiter have been predicted for centuries for many scientific purposes. First BDL and IMCCE (from 1998) used to send these predictions to most of the ephemerides services worldwide after official agreements. The mutual events have been predicted in the past (Arlot 1973, 1982, 1984, 1990, 1996), and more recently with higher accuracy (Arlot 2002, 2008; Emel'yanov et al. 1994; Emelianov 1996c; Emel'yanov 2002). Due to the sensitivity to ephemerides'accuracy and to the computing method, we provide on the MULTI-SAT server several different predictions by several authors.

Links to other useful ephemerides are also available:

- special ephemerides of the coorbital satellites of Saturn for infrared observations;

- apparent encounters of the outermost satellites of Jupiter;

- apparent encounters of the stars Tycho-2 Catalogue and main natural satellites. 
Links to other services useful for ephemerides purposes are also available such as the ephemerides for physics observations of the Solar System bodies (software MOVIS).

All the web pages of the MULTI-SAT ephemerides have links to explanations and to the parameters of the satellites.

\section{Theories and methods used for calculating the ephemerides of all natural planetary satellites}

We list below the theories and methods used in our programs to calculate the ephemerides of all natural planetary satellites.

\subsection{Martian satellites (Phobos and Deimos)}

Three analytical theories are used and proposed to the user:

- the analytical theory developed by Chapront-Touze (Chapront-Touze 1990) from the observations of the catalog by Morley and from the observations made at Pic du Midi in 1988 (Colas 1997);

- the analytical theory built by Kudryavtsev (Kudryavtsev et al. 1997). This is one of the most precise analytical theories based on all ground-based observations and observations made by Viking-1, Viking-2, Mariner, and Phobos-2 spacecrafts, which had been carried out until 1990;

- the brand new theory published by Lainey (2007), built for the Mars Express project based upon a numerical integration and fitted to all the observations available in the database.

\subsection{Satellites of Jupiter}

\subsubsection{Galilean satellites ( 11 to $\mathrm{J} 4$ )}

In our server, we propose a choice between three models:

- the G-5 ephemerides based upon Lieske's work and fitted on photographic observations by Arlot (1982);

- the E-5 ephemerides similar to G-5 but more recently made by Lieske (1998);

- the L1 ephemerides based upon a brand new theory by Lainey (2004).

For the interval 1800-2000, the Lainey's theory may be considered to be more precise than that of Lieske because more complete database of observations was used and better modeling.

\subsubsection{Inner satellites (J5, J14, J15, and J16)}

We use simple original models of motion based on precessing Keplerian ellipse.

\subsubsection{Outer satellites (J6 to J13)}

For outer Jovian satellites, J6-J13, the original numerical models of motion described in (Emel'yanov 2005) are used. Ephemerides of these satellites are based on all published ground-based observations made since 1905. The ephemerides may be calculated with our ephemeride server for the interval 1905-2025. We update the models of satellite motion and improve ephemerides precision as new observations become available.

\subsection{Satellites of Saturn}

\subsubsection{Major satellites (S1 to S8)}

Three analytical theories have been proposed:

- the theory of Harper and Taylor (Harper \& Taylor 1993; Taylor et al. 1987) that was realized as a calculating program using formulae given in the paper itself;

- the theory of Dourneau Do93 (Dourneau 1987, 1993);

- the theory TASS 1.7 of Vienne \& Duriez (Vienne \& Duriez 1995; Duriez \& Vienne 1997). This theory was adapted for the ephemerides service using the author's version of the calculating program. The TASS 1.7 theory should be considered as more reliable and precise because it is based on a bigger number of observations and takes into account more perturbation factors influencing the motion of the satellites. Comparison of this theory with the observations made in past years reveals that it agrees with them better than with other theories.

\subsubsection{Small coorbital satellites ( $\$ 12$ Helene, $\mathrm{S} 13$ Telesto, S14 Calypso) moving in orbits of the major satellites (Lagrangians)}

Our ephemerides service uses a version of the analytical theory of these satellites published in the paper of Oberti \& Vienne (2003).

\subsubsection{Inner satellites of Saturn (S15 to S18, S35)}

For these satellites located inside the orbit of Mimas, we used the model of the precessing ellipses. The ephemerides are realized for two versions of orbital parameters, the first taken from Bosh \& Rivkin (1996), French et al. (2000), Showalter (1991). These parameters are determined from ground-based observations. Second version of orbital parameters is determined from the observations made by the spacecraft Cassini (Porco et al. 2005).

\subsubsection{New small inner satellites (S32 Methone, S33 Pallene, S34 Polydeuce)}

The ephemerides are realized with the version of orbital parameters determined from the observations made by the spacecraft Cassini (Porco et al. 2005).

\subsubsection{Coorbital satellites, S10 Janus and S11 Epimetheus}

We have programmed formulae and the set of orbital parameters published in papers of Yoder et al. (1989) and Nicholson et al. (1992). There are only a few observations of these satellites. The theory being used agrees well with the observations.

\subsubsection{Satellite S9 Phoebe}

The new ephemeris of Phoebe, based on 1606 ground-based observations made at 39 observatories in the 103 year time interval, was elaborated in Emel'yanov (2007). The ephemeris will be updated as new observations become available. 


\subsection{Satellites of Uranus}

\subsubsection{Major satellites (U1 to U5)}

Actually there is only one analytical theory for these satellites, which was published in such a manner that it can be used to calculate ephemerides. This is the theory of Laskar \& Jacobson (Laskar \& Jacobson 1987) based on observations made from 1911 to 1986 . The authors' version of a calculating program was adapted for our ephemerides server. We will soon add new models made more recently, such as LA06 (Arlot et al. 2006).

\subsubsection{Inner satellites (U6 to U15 and U25 to U27)}

For these satellites we used the model of the precessing ellipses. Orbital parameters were taken from the papers of Jacobson (1998) and Showalter \& Lissauer (2006). Mean motions of the eight inner satellites of Uranus were slightly corrected using the paper by Pascu et al. (1998).

\subsection{Satellites of Neptune}

\subsubsection{N1 Triton}

For this satellites we used the model of the precessing ellipse built by Jacobson (1991). The precision of the ephemerides obtained using these data is the same as for ground-based observations.

\subsubsection{N2 Nereid}

For this satellite we used our original model built from observations with numerical integration of the equations of motion.

\subsubsection{Inner satellites (N3 to N8)}

For these satellites we used the model of the precessing ellipses. Orbital parameters were taken from the paper by Owen et al. (1991). Mean motions and semi-major axes of the orbits of the four inner satellites of Neptune were slightly corrected using values from the paper of Pascu et al. (2004).

\subsection{Outer satellites of Jupiter, Saturn, Uranus and Neptune discovered after 1997}

From 1997 to July 200746 outer satellites of Jupiter, 37 outer satellites of Saturn, 9 outer satellites of Uranus, 6 outer satellites of Neptune were discovered. For these new outer satellites of planets, the original models of motion were elaborated. These models are defined from all published ground-based observations with the numerical integration of equations of motion (Emel'yanov \& Kanter 2005). Our ephemerides of the new outer satellites of planets are based on these models of motion.

The ephemerides will be updated as new observations become available.

\subsection{Satellites of Pluto (P1 to $P 3)$}

To calculate the ephemerides of these satellites we used the model of Keplerian motion with parameters taken from the paper of Tholen \& Buie (1997) for Charon and from the paper of Buie et al. (2006) for Nix and Hydra.

The theories and methods used in our ephemerides service are steadily updated as new, more precise, versions of these theories appear in publications. Our original models of motion of outer planetary satellites are also improved as new observations are made.

\section{Planetary ephemerides used in the software}

To calculate topocentric or geocentric satellite coordinates, the heliocentric rectangular coordinates of the corresponding planet are necessary. To obtain them, we used numerical ephemerides of the planets and the Moon DE200/LE200 (Standish 1990) and updated versions of these ephemerides (DE405/LE405, DE406/LE406). The planetary ephemerides VSOP87 (Bretagnon \& Francou 1988) are also included in our ephemerides service, as well as recently published INPOP ephemerides (Fienga et al. 2008). The user may select any of these planetary ephemerides. For the time intervals for which the selected planetary ephemerides were not calculated, our calculating program uses the analytical theory of planetary motion based on the works of Newcomb, Hill, Goffin, Meyes, and Stuart. In this case the calculating programs that we use are given in the book of Montenbruck \& Pfleger (2000) where the corresponding references may be found. In this case, absolute satellite coordinates are obtained with low precision. However, such ephemeris does not influence the precision of relative coordinates too much (planet-satellite, satellite-satellite).

\section{Precision and accuracy of the ephemerides}

Even if done with great care, the ephemerides still present differences from the real positions of the bodies. This stems from several facts. The construction of the satellite ephemerides consists of several steps. Differential equation of motions are composed first. The errors are introduced at this stage, since some small perturbing forces are neglected, and the physical parameters of satellites entering the equations are known with limited accuracy. Equation of motions are then solved, i.e., the coordinates of satellites as functions of time and orbital parameters are obtained. Any method for integrating the equation of motions is always approximate, thereby introducing additional errors. All these errors determine the precision of the theoretical model of the satellite motion on the assumption that the orbital parameters are assigned exactly. We call this the internal precision of the theoretical model.

In a second step one fits the orbital parameters of satellites to the observations, so a model of the satellite motion is obtained, from which the ephemerides are then calculated at any given moments of time. Since the observations are inaccurate, additional errors are introduced into the ephemerides.

After the fit of the orbital parameters to the observations, the deviation of the observed position from the calculated one is determined at each moment of observation, i.e., O-C. This deviation is the sum of the error of the theoretical model and the observation error. The rms of $\mathrm{O}-\mathrm{C}$ through all these observations is called the accuracy of the model of the satellite motion or external accuracy. However, this external accuracy is calculated only on the interval of time on which the observations were made. In fact, the user is more interested by the accuracy of the ephemerides outside this interval, mainly in the future. The accuracy then depends on the propagation of the errors in the extrapolation of the ephemerides, i.e. on the following factors: the accuracy of theoretical model (internal precision), the accuracy of observation, the time interval of observations, the distribution of observations upon the time, the distribution of observations 
Table 1. Accuracy of the ephemerides of the Martian satellites in mas.

\begin{tabular}{cccccc}
\hline \hline Model & Satellite & $\begin{array}{c}\text { Internal } \\
\text { precision }\end{array}$ & $\begin{array}{c}\text { External } \\
\text { accuracy }(1 \sigma)\end{array}$ & $\begin{array}{c}\text { Observational } \\
\text { period }\end{array}$ & $\begin{array}{c}\text { Accuracy of the ephemerides on the } \\
\text { period 1988-2017 (max deviation) }\end{array}$ \\
\hline Lainey & Phobos & 0.1 & 3 & $1877-2005$ & 24 \\
& Deimos & 0.1 & 3 & $1877-2005$ & 60 \\
\hline Kudryavtsev (1997) & Phobos & 0.6 & 6 & $1877-1988$ & 60 \\
& Deimos & 0.6 & 24 & $1877-1988$ & 600 \\
\hline Chapront-Touzé & Phobos & 0.5 & 10 & $1877-1988$ & 600 \\
& Deimos & 3 & 30 & $1877-1988$ & 1000 \\
\hline
\end{tabular}

Table 2. Accuracy of the ephemerides of the Galilean satellites in mas.

\begin{tabular}{cccccc}
\hline \hline Model & Satellite & $\begin{array}{c}\text { Internal } \\
\text { precision }\end{array}$ & $\begin{array}{c}\text { External } \\
\text { accuracy }(1 \sigma)\end{array}$ & $\begin{array}{c}\text { Observational } \\
\text { period }\end{array}$ & $\begin{array}{c}\text { Accuracy of the ephemerides on the } \\
\text { period 1985-2017 (max deviation) }\end{array}$ \\
\hline Lainey L1 & J1 & 3 & 60 & $1891-2003$ & 25 \\
& J2 & 8 & 60 & $1891-2003$ & 40 \\
& J3 & 6 & 60 & $1891-2003$ & 50 \\
& J4 & 13 & 60 & $1891-2003$ & 50 \\
\hline Arlot G5 & J1 & 3 & 90 & $1891-1978$ & 120 \\
& J2 & 3 & 100 & $1891-1978$ & 120 \\
& J3 & 3 & 100 & $1891-1978$ & 120 \\
\hline
\end{tabular}

Table 3. Accuracy of the ephemerides of the major satellites of Saturn in mas.

\begin{tabular}{cccccc}
\hline \hline Model & Satellite & $\begin{array}{c}\text { Internal } \\
\text { precision }\end{array}$ & $\begin{array}{c}\text { External } \\
\text { accuracy }(1 \sigma)\end{array}$ & $\begin{array}{c}\text { Observational } \\
\text { period }\end{array}$ & $\begin{array}{c}\text { Accuracy of the ephemerides on the } \\
\text { period 1977-2017 (max deviation) }\end{array}$ \\
\hline TASS & S1 & 3 & 90 & $1877-1992$ & 50 \\
& S2 & 3 & 70 & $1877-1992$ & 50 \\
& S3 & 3 & 70 & $1877-1992$ & 50 \\
& S4 & 3 & 70 & $1877-1992$ & 50 \\
& S5 & 3 & 70 & $1877-1992$ & 50 \\
& S6 & 3 & 70 & $1877-1992$ & 50 \\
& S7 & 30 & 150 & $1877-1992$ & 50 \\
& S8 & 10 & 150 & $1877-1992$ & 50 \\
\hline Do93 & S1 & 200 & 180 & $1886-1985$ & 300 \\
& S2 & 27 & 150 & $1886-1985$ & 90 \\
& S3 & 48 & 150 & $1886-1985$ & 60 \\
& S4 & 55 & 150 & $1886-1985$ & 80 \\
& S5 & 60 & 120 & $1886-1985$ & 90 \\
& S6 & 60 & 130 & $1886-1985$ & 1800 \\
& S7 & 100 & 200 & $1886-1985$ & 700 \\
\hline
\end{tabular}

along the orbit of satellite, the time interval between the last observation and the moment at which the ephemeris is determined. For this reason the accuracy of ephemerides outside the interval of observations can be considerably worse than internal precision or external accuracy. It is very difficult to determine the real accuracy of ephemeris at any time. Attempts at theoretical studies do not lead to the reliable estimations. Some estimations of accuracy can be obtained in practice by comparing several ephemerides independently made or (better) by comparison to new observations made outside the interval of time where the ephemerides were fit.

Let us examine an example showing the real errors of the ephemeris, which considerably exceed the external accuracy. The model of the motion of the satellite of Saturn Prometheus (French et al. 2000) on the basis of ground-based observations, have an external accuracy of 0.03 arcsec. The model of the motion of this satellite from the Cassini spacecraft obsertvations (Porco at al. 2005) has external accuracy of 0.05 arcsec. The comparison of the ephemeris of satellite Prometheus in the time interval of 30 days from 1 January 2007, obtained on the basis of two independent models of motion, shows the disagreements that exceed 2 arcsec. This is considerably more than external accuracy.

We give in Tables 1 to 5 the precision of the theoretical model of motion (internal precision) for the different groups of satellites and the accuracy of the agreement of the theory with the observations used for the fit of the model (external accuracy), together with the period of the observations.

For the user of the ephemerides server MULTI-SAT, it is important to know the maximum ranges of the ephemeris errors. There is no reliable method of evaluating the ephemeris precision outside the observational time interval. Therefore in Tables 1 to 5 we give only approximative estimates of the ephemerides accuracies in geocentric astrometric relative coordinates of the satellites using some comparisons of the ephemerides with observations and also different theories between them. One should consider that the external accuracy was calculated as rms $(\sigma)$ of the $\mathrm{O}-\mathrm{C}$, but maximum deviations are given in our estimations of the ephemerides accuracy. 
Table 4. Accuracy of the ephemerides of the major satellites of Uranus in mas.

\begin{tabular}{lccccc}
\hline \hline Model & Satellite & $\begin{array}{c}\text { Internal } \\
\text { precision }\end{array}$ & $\begin{array}{c}\text { External } \\
\text { accuracy }(1 \sigma)\end{array}$ & $\begin{array}{c}\text { Observational } \\
\text { period }\end{array}$ & $\begin{array}{c}\text { Accuracy of the ephemerides on the } \\
\text { period 1977-2017 (max deviation) }\end{array}$ \\
\hline LA06 & S1 & 1 & 60 & $1954-2005$ & 40 \\
& S2 & 3 & 60 & $1954-2005$ & 40 \\
& S3 & 7 & 60 & $1954-2005$ & 40 \\
& S4 & 7 & 60 & $1954-2005$ & 40 \\
& S5 & 1 & 60 & $1954-2005$ & 60 \\
\hline LJ86 & S1 & 1 & 130 & $1911-1986$ & 60 \\
& S2 & 3 & 130 & $1911-1986$ & 60 \\
& S3 & 7 & 130 & $1911-1986$ & 60 \\
& S4 & 7 & 130 & $1911-1986$ & 60 \\
\hline
\end{tabular}

Table 5. Accuracy of the ephemerides of some outer satellites of Jupiter, Saturn, and Neptune in mas.

\begin{tabular}{ccccc}
\hline \hline Satellite & $\begin{array}{c}\text { Internal } \\
\text { precision }\end{array}$ & $\begin{array}{c}\text { External } \\
\text { accuracy }(1 \sigma)\end{array}$ & $\begin{array}{c}\text { Observational } \\
\text { period }\end{array}$ & $\begin{array}{c}\text { Accuracy of the ephemerides on the } \\
\text { period 2007-2017 (max deviation) }\end{array}$ \\
\hline J6 & 12 & 630 & $1905-2003$ & 150 \\
J7 & 12 & 670 & $1905-2003$ & 150 \\
J8 & 12 & 640 & $1908-2003$ & 150 \\
J9 & 12 & 760 & $1914-2003$ & 150 \\
J10 & 12 & 630 & $1938-2003$ & 200 \\
J11 & 12 & 670 & $1938-2003$ & 250 \\
J12 & 12 & 520 & $1951-2003$ & 300 \\
J13 & 12 & 480 & $1974-2003$ & 150 \\
\hline S9 Phoebe & 8 & 240 & $1904-2007$ & 300 \\
\hline N2 Nereid & 2 & 380 & $1949-2006$ &
\end{tabular}

For the coorbital satellites of Saturn (Helene, Telesto, Calypso, Janus, and Epimetheus), the ephemerides accuracy is of about 0.4 arcsec on the time interval from 1980 to 2017. The ephemerides of Triton (satellite of Neptune) were calculated with the accuracy of 0.1 arcsec on the time inteval from 1800 to 2017.

For the inner satellites of Jupiter, Uranus, and Neptune the ephemeris accuracy is about 0.3 arcsec on the time interval 1980 to 2017 . On the observational time intervals, the ephemeris accuracy for these satellites may be somewhat better. The precision of the ephemerides of satellite of Pluto Charon on the time interval from 1970 to 2017 is not worse than 0.1 arcsec.

The ephemerides of the outer satellites of Jupiter, Saturn, Uranus, and Neptune discovered after 1997 are based on the observations made on very short time intervals. For some satellites, the intervals of observations cover a small part of one orbital period. Therefore the ephemerides of these satellites cannot be reliable. On the time intervals of observations, the precision the ephemerides is of about 0.5 arcsec. Outside these time intervals, the errors of the ephemerides may be more than 10 arcsec. Analysis of Avdyushev \& Banshchikova (2007) shows that the errors of the ephemerides of some new outer satellites can be close to the dimensions of their orbits.

Note that the ephemerides of all outer satellites of Jupiter, Saturn, Uranus and Neptune are calculated with our numerical model of motion only on the time intervals from the time moment of first published observation up to 2027. Outside of these time intervals, we use Keplerian orbits with some versions of orbital parameters. The Keplerian orbits may only give very approximative ephemerides. For some satellites the estimations of the real accuracy of ephemeris remain unknown.

The accuracy of the ephemerides is better for objects known for years and subject to research for a long time. For example, the Galilean satellites have been known since 1610, and even if their motions are very complicated, the ephemerides are quite good.

With an analytical theory or precessing ellipse model, our software calculates the ephemerides of natural satellites on the time interval from 1000 to 3000 . But with the best precision, the ephemerides are only available for the time moments running not far from the observational interval.

The accuracy of the ephemerides of natural satellites decreases as the time elapsed since the period of observation increases. Continuing observations are needed to monitor the ephemerides and, when significant discrepancies occur, an update of ephemerides is needed.

In most cases the errors of ephemerides are dominant in the orbital longitude. Only if the time interval of observation covers a small part of orbital period may the errors be as large across the trajectory of satellite as along it.

When the absolute coordinates (right ascension and declination) are calculated, the error of a natural satellite ephemeris is the sum of the error of planetary ephemeris and the error of satellites motion model. In our estimates of the precision of the natural satellites ephemerides, we only discuss the precision of the astrometric coordinates of the satellite relative to the planet or another satellite.

\section{Conclusions}

A new facility for the production of the ephemerides of all natural satellites of planets (except the Moon) has been created in IMCCE and SAI. Ephemerides are the final result of research as they are based on the complete knowledge about dynamics of the satellites of planets including all available observational data.

For all outer planetary satellites, original numerical models of motion are used that are based on all published observations. 
For other satellites, the theoretical models of the motion are taken from the most recent publications.

The access to the elaborated software for the ephemerides of natural satellites is organized on the webpages of the so-called server MULTI-SAT, which is available on the Internet. The sites URL are http: //www . imcce. fr/sat (IMCCE) and http: // www. sai.msu.ru/neb/nss/index.htm (SAI).

Simple interface of the software allows easy implantation into a virtual observatory or other web-service.

Acknowledgements. This work was made possible thanks to the "Programme International de Cooperation Scientifique" No. 3840 of CNRS and the Russian Foundation for Basic Research, project No. 07-02-92169-CNRS-a.

\section{References}

Arlot, J.-E. 1973, L'Astronomie, 87, 289

Arlot, J.-E. 1982, A\&A, 107, 305

Arlot, J.-E. 1984, A\&A, 107, 305

Arlot, J.-E. 1990, A\&A, 237, 290

Arlot, J.-E. 1996, A\&A, 138, 113

Arlot, J.-E. 2002, A\&A, 383, 719

Arlot, J.-E. 2008, in press

Arlot, J.-E., \& Emel'yanov, N. V. 2008, in press

Arlot, J.-E., Lainey, V., \& Thuillot, W. 2006, A\&A, 456, 1173

Avdyushev, V. A., \& Banshchikova, M. A. 2007, Sol. Syst. Res., 41, 413

Bosh, A. S., \& Rivkin, A. 1996, Science, 272, 518

Bretagnon, P., \& Francou, G. 1988, A\&A, 202, 309

Buie, M. W., Grundy, W. M., Young, E. F., et al. 2006, AJ, 132, 290

Chapront-Touze, M. 1990, A\&A, 240, 159

Colas, F. 1992, A\&AS, 96, 485

Dourneau, G. 1987, Ph.D. Thesis

Dourneau, G. 1993, A\&A, 267, 292

Duriez, L., \& Vienne, A. 1997, A\&A, 324, 366

Emelianov, N. V. 1996a, Ephemerides software of natural satellites, Annales de physique, PHESAT95 Workshop, 21, C1-41

Emel'yanov, N. V. 1996b, Astron. Lett., 22, 135
Emelianov, N. V. 1996c, Sol. Syst. Res., 30, 366

Emel'yanov, N. V. 2002, Sol. Syst. Res., 36, 353

Emel'yanov, N. V. 2005, A\&A, 435, 1173

Emel'yanov, N. V. 2007, A\&A, 473, 343

Emel'yanov, N. V., \& Kanter, A. A. 2005, Sol. Syst. Res., 39, 112

Emel'yanov, N. V., Gasanov, S. A., \& Nasonova, L. P. 1994, Astron. Rep., 38, 708

Emel'yanov, N. V., Arlot, J.-E., Varfolomeev, M. I., et al. 2006, Cosmic Res., 44, 128

Fienga, A., Manche, H., Laskar, J., \& Gastineau, M. 2008, A\&A, 477, 315

French, R. G., McGhee, C., Dones, L., \& Lissauer, J. 2000, Am. Astron. Soc., DPS meeting, 32, 49.12

Harper, D., \& Taylor, D. B. 1993, A\&A, 268, 326

Jacobson, R. A. 1998, AJ, 115, 1195

Jacobson, R. A., Riedel, J. E., \& Taylor, A. H. 1991, A\&A, 247, 565

Kudryavtsev, S. M., Kolyuka, Y. F., \& Tikhonov, V. F. 1997, New Analytical Theory of Motion of Phobos and Deimos for Navigation Support of Mission to Mars, Proceedings of the 12th International Symposium on Space Flight Dynamics, ESA SP-403, 377

Lainey, V., Vienne, A., \& Duriez, L. 2004, A\&A, 420, 1171

Lainey, V., Dehant, V., \& Patzold, M. 2007, A\&A, 465, 1075

Laskar, J., \& Jacobson, R. A. 1987, A\&A, 188, 212

Lieske, J. H. 1998, A\&AS, 129, 205

Montenbruck, O., \& Pfleger, T. 2000, Astronomy on the personal computer (Berlin, Heidelberg: Springer-Verlag)

Nicholson, P. D., Hamilton, D. P., Matthews, K., \& Yoder, C. F. 1992, Icarus, 100,464

Oberti, P., \& Vienne, A. 2003, A\&A, 397, 353

Owen, W. M. Jr., Vaughan, R. M., \& Synnott, S. P. 1991, AJ, 101, 1511

Pascu, D., Rohde, J. R., Seidelmann, P. K., et al. 1998, AJ, 115, 1190

Pascu, D., Rohde, J. R., Seidelmann, P. K., et al. 2004, AJ, 127, 2988

Porco, C. C., Baker, E., Barbara, J., et al. 2005, Science, 307, 1226

Showalter, M. R. 1991, Nature, 351, 709

Showalter, M. R., \& Lissauer, J. J. 2006, Science, 311, 5763, 973

Standish, E. M. 1990, A\&A, 233, 252

Taylor, D. B., Sinclair, A. T., \& Message, P. J. 1987, A\&A, 181, 383

Tholen, D. J., \& Buie, M. W. 1997, Icarus, 125, 245

Vienne, A., \& Duriez, L. 1995, A\&A, 297, 588

Yoder, C. F., Synnott, S. P., \& Salo, H. 1989, AJ, 98, 1875 